\title{
Burkitt Lymphoma in children causing an osteolytic lesion in the mandible: A case report
}

Wouter De Coninck ${ }^{1}$, Dries Govaerts ${ }^{1}$, Michel Bila ${ }^{1}$, Griet Vansteenkiste ${ }^{1}$, Anne Uyttebroeck $^{1}$, Thomas Tousseyn ${ }^{2}$, and Constantinus Politis ${ }^{1}$

${ }^{1} \mathrm{KU}$ Leuven

${ }^{2} \mathrm{KU}$ Leuven University Hospitals Leuven

November 2, 2020

\begin{abstract}
Burkitt lymphoma is a rapidly progressive disease which requires imaging, biopsy, prompt diagnosis and early aggressive chemotherapeutic treatment. We discuss a 7-year-old girl presenting with a persistent and painful unilateral swelling of the jaw. Lymphomas typically manifest as poor defined osteolytic lesions radiographically, as is presented in this case.
\end{abstract}

Burkitt Lymphoma in children causing an osteolytic lesion in the mandible: A case report

Short Title : Osteolytic lesions in Burkitt Lymphoma

Wouter DE CONINCK, MD ${ }^{1,2}$; Dries GOVAERTS, MD, DDS ${ }^{1,2}$; Michel BILA, MD, DDS ${ }^{1,2}$; Griet VANSTEENKISTE, DDS ${ }^{3,4}$; UYTTEBROECK Anne, $\mathrm{MD}, \mathrm{PhD}^{5,6}$; Thomas TOUSSEYN, MD, $\mathrm{PhD}^{7}$; Constantinus POLITIS, MD, DDS, MM, MHA, $\mathrm{PhD}^{1,2}$

1 OMFS-IMPATH Research Group, Department of Imaging and Pathology, Faculty of Medicine, Catholic University Leuven, Leuven, Belgium

2 Department of Oral and Maxillofacial Surgery, University Hospitals Leuven, Leuven, Belgium

3 Department of Oral Health Sciences, KU Leuven, Leuven, Belgium

4 Department of Pediatric Dentistry, University Hospitals Leuven, Leuven, Belgium

5 Department of Oncology, KU Leuven, Leuven, Belgium

6 Department of Pediatric Hematology and Oncology, University Hospitals Leuven, Leuven, Belgium

7 Department of Imaging and Pathology, University Hospitals Leuven, Leuven Cancer institute, Leuven, Belgium

Word count: 1578

Corresponding author

Constantinus Politis

Department of Oral and Maxillofacial Surgery,

University Hospitals Leuven,

Kapucijnenvoer 33 Leuven, 
3000 Belgium.

Telephone number: +3216332464

Fax number: +3216332437

E-mail: constantinus.politis@uzleuven.be

Burkitt Lymphoma in children causing an osteolytic lesion in the mandible: A case report

\section{ABSTRACT}

Burkitt lymphoma is a rapidly progressive disease which requires imaging, biopsy, prompt diagnosis and early aggressive chemotherapeutic treatment. We discuss a 7-year-old girl presenting with a persistent and painful unilateral swelling of the jaw. Lymphomas typically manifest as poor defined osteolytic lesions radiographically, as is presented in this case.

Key words: Orthopantomography; Panoramic radiograph; Burkitt Lymphoma; Osteolytic lesions; Pediatrics; Case report

\section{KEY CLINICAL MESSAGE}

Imaging is the first step in diagnosing a persistent swelling of the jaw. A lymphoma in the jaw typically manifests as a poor defined osteolytic lesion. A biopsy is mandatory and will result in definite diagnosis.

\section{INTRODUCTION}

Burkitt lymphoma (BL) is a highly aggressive B cell non Hodgkin lymphoma (NHL) which manifests in 3 different clinical forms: the endemic form, the sporadic form and the immunodeficiency associated form. ${ }^{1-3}$

They are histologically identical but there are differences in epidemiology, clinical presentation and genetic characteristics. ${ }^{2}$ The endemic variant is most prevalent in Africa due to chronic Epstein-Barr virus (EBV) and malaria. ${ }^{4,5}$ The sporadic form is most observed in the Western world. In the developed world BL accounts for about $80 \%$ of all B-cell NHL in children. ${ }^{2-4,6}$ The immunodeficiency associated variant is mostly seen in patients with AIDS or other immune compromising disorders. ${ }^{2}$

The endemic form involves the jaw in over $50 \%$ of cases. It often manifests as a painless facial mass that spreads to extra nodal foci. ${ }^{2,7}$ The sporadic form commonly comes with a bulky abdominal mass. ${ }^{5,8}$ Oral lesions are presented in $9 \%$ of the cases of the sporadic form. ${ }^{9}$ Adenopathies and bone marrow involvement are present more often in the immunodeficiency associated form, with the clinical presentation similar to that of the sporadic form of BL. ${ }^{8}$

Focusing on oral presentation, the mandible is the most frequent location. The posterior part of the jaw is the most affected area. ${ }^{7}$ Swelling, pain, dental displacements and facial asymmetry are the main findings in oral BL. ${ }^{10}$ Other reported symptoms are increased tooth mobility and tooth pain because of infiltration in the pulp, especially by developing teeth. ${ }^{7}$ Paresthesia of the inferior alveolar nerve or other sensory facial nerves is common. ${ }^{10}$

\section{CASE PRESENTATION}

A 7-year-old girl presented at her local maxillofacial department with pain on the left side of the mandible. Two months before presentation these complaints began as intermittent pain. The left jaw began to swell and ache intensively. The diagnosis of inflammation was most probable given the young age, pain and the localized swelling. ${ }^{11}$ Before any imaging, the local maxillofacial surgeon tried to treat a possible abscess by incision combined with intravenous (IV) amoxicillin clavulanic acid. Only significant edema of the soft tissue without pus drainage was recorded. Superficial necrosis was noticed due to friction of the upper molars in the left cheek (Figure 1A). The patient had no medical history. A referral to the university hospital took place. 
After panoramic radiograph (Figure 2) further imaging modalities were initiated based on osteolytic lesions found in the lower left quadrant. Absence of the thin circumferential cortical layer around the second molar in the lower jaw on the left side was recorded. ${ }^{12}$ Through ultrasound imaging an inhomogeneous, relatively welldefined soft tissue mass was shown above the submandibular salivary gland with invasion in the mandible. A computed tomography (CT) (Figure 3C) was performed which showed a mass in the left masseter muscle region, fixed to the ascending mandibular ramus with osteolysis of the bony tissue. These findings were confirmed using magnetic resonance imaging (MRI)(Figure 3B). For staging a positron emission tomography - computed tomography (PET-CT)(Figure 3A) was conducted indicating metastases which were confirmed through ultrasonography of the abdomen.

A final diagnosis was made by biopsy and histopathological examination respectively. ${ }^{13}$ A partial resection of the mass that interfered with the occlusion was performed. The histopathological examination revealed an ulcerated fragment mucosa with the presence of a poorly differentiated neoplastic process, consisting of a diffuse proliferation of medium-sized, monomorphic blastoid cells with round vesicular to hyperchromatic nuclei. The nuclei contained small nucleoli and numerous mitotic figures were present. Between the cells there are tingible body macrophages giving rise to a starry sky appearance. (Figure 4) The immunophenotype identified the presence of a B-cell lymphoproliferative process expressing CD20, CD10, BCL6, MYC and a proliferation rate of $100 \%$, but no expression of BCL2, TDT, cytokeratin, desmin, myogenin, CD99, MPO, CD3 or EBV. PCR showed monoclonal immunoglobulin heavy and light chain rearrangements. Fluorescence in-situ hybridization (FISH) confirmed diagnosis of Burkitt Lymphoma (MYC rearrangement, stage III, $\mathrm{LDH}>2 \mathrm{~N}$, high risk group $)^{14}$ resulting in an overproduction of the MYC protein due to genetic rearrangement. The involvement of adenopathies on both sides of the diaphragm on PET-CT defines it as a stage III lesion based on the St. Jude classification. There was no invasion of the bone marrow. The $\mathrm{LDH}$ concentration was $786 \mathrm{U} / \mathrm{L}$ (normal range $192-321 \mathrm{U} / \mathrm{L}$ ) indicating highly proliferative disease. Risk stratification was performed for treatment planning and is linked to the stage of the disease, site as well as LDH level. ${ }^{13-15}$ Chemotherapy was initiated combined with rituximab according to the intergroup B-NHL Ritux 2010 trial group B at the department for pediatric oncology and hematology. ${ }^{14}$

During hospitalization sufficient pain relief was achieved with IV administration of paracetamol and tramadol by weight and oral hygiene was restarted subsequently. After a part of the chemotherapy was administered the swelling diminished significantly. (Figure 1B)

\section{DISCUSSION}

The diagnostic approach of a facial swelling in a child is always a source of difficulties for the clinician. Especially for a disease with such heterogenous clinical symptoms as BL. ${ }^{16}$ One must be aware of pathology other than dental infection when cortical bone is absent. BL has a predominance in males, mostly in young patients. $., 7,10,17$

The panoramic radiograph (Figure 2) shows the dentition in transition from deciduous to permanent. The second molar of each quadrant is in development given the incomplete root formation. ${ }^{18}$ The thin circumferential cortical layer around the second molar in the lower jaw on the left side, which normally presents as the typical radiological finding of the dental sac, is absent. ${ }^{12}$ This aspect of this panoramic radiograph could be described as an osteolytic zone in the lower left quadrant at the level of the angle and ramus of the mandible. This radiolucency at dental level has already been described in a case with leukemia by Curtis et al. presenting the absence of the lamina dura. ${ }^{19}$ A similar dental osteolytic presentation of oral BL has been reported by Hanazawa et al. and Wood et al. ${ }^{20,21}$ The dental sac embryologically relates to the periodontal ligament which closely interacts with the lamina dura of the alveolar bone. ${ }^{18,22,23}$ Radiographically BL may begin as multiple lesions, which later merge into a larger radiolucency with an expansile behavior. The lesions are radiolucent in almost all cases, particularly in the jaw of a child. ${ }^{10}$

Other osteolytic pathology should be taken into account in the differential diagnosis. Metastatic neuroblastoma, Ewing's tumor, rhabdomyosarcoma, osteolytic osteosarcoma and other NHL lymphomas/leukemia must be considered. 
The initial approach should involve dental imaging due to easy accessibility and the value to exclude an odontogenic origin, one of the most prevalent causes of facial swelling in children. ${ }^{11}$ In case no odontogenic origin could be found, ultrasonography should be performed, especially in case of nodules in the neck. ${ }^{5}$ For NHL, extra nodal involvement occurs at a frequency of $20-30 \%$ in the head and neck region. ${ }^{13}$ In order to identify bone resorption and the precise radiologic extent of the disease both CT and MRI are valuable investigations. CTs have better image quality for bone imaging and a lower cost. MRI doesn't use ionizing radiation, has a better characterization of bone marrow and is superior in soft tissue contrast. ${ }^{5}$ PET-CT is useful for functional and anatomic assessment during tumor staging and tumor response evaluation. ${ }^{5}$

Several studies strongly suggest the involvement of the EBV in BL, since EBV inhibits cell death and contributes to the development and maintenance of BL. ${ }^{7}$ At the molecular level the deregulation of the MYC proto-oncogene is a characteristic feature of BL. ${ }^{24}$

The therapy of choice for all types of BL are short, intensive short courses of a combination of different types of chemotherapy (cyclophosphamide, vincristine, prednisone, doxorubicin, alkylators and etoposide).2,6,14 The addition of immunotherapy (rituximab) to chemotherapy is an effective therapeutic option in highrisk, high-grade, mature BL which increases the overall survival significantly. ${ }^{14}$ New studies are now being enrolled to define the therapeutic-toxic margins of current therapy without further increasing the risk of relapsing disease. Often the immune therapy is started in the second cycle to minimize adverse effects. ${ }^{2}$ There is no need for surgical resection nor for radiotherapy because of the high sensitivity of BL cells to chemotherapy and an increased rate of local complications correlated with early surgical interventions. ${ }^{2,25}$ Surgical excision has only a prognostic benefit when almost-complete resection can be performed without delaying chemotherapy. ${ }^{26}$ In this case total resection was not achieved but the hinder for the patient's occlusion was the incentive.

Prognostic factors are lactate dehydrogenase (LDH) concentrations, stage of disease, leukemic bone marrow and central nervous system (CNS) involvement together with treatment-related factors such as late or incomplete response. ${ }^{14}$

The overall survival rate for standard risk, early/moderate stage BL with chemotherapy alone is about 97 - 98\%. ${ }^{14}$ Where the prognosis for the more advanced, higher risk stages is $87,3 \% .{ }^{14} \mathrm{~A}$ recent randomized, phase III trial found that for these high-risk, high-stage BL the addition of six cycles of rituximab to the standard chemotherapy resulted in an overall survival of more than $95 \%{ }^{14}$

Follow-up is performed every two to three months the first year following therapy through clinical investigations, abdominal ultrasonography and blood investigations. The frequency of follow-up decreases in the subsequent years. Most relapses occur in the first year following therapy. ${ }^{14}$

\section{CONCLUSION}

$\mathrm{BL}$ is considered to have an aggressive behavior and moderate overall survival rate. ${ }^{17}$ Imaging is the first step in diagnosing a persistent swelling of the jaw. A lymphoma in the jaw typically manifests as a poor defined osteolytic lesion. The absence of the radiographical signs of the dental follicle of a germinal tooth is presented in this case. A biopsy is mandatory and will result in definite diagnosis. ${ }^{10,27}$

\section{CONFLICTS OF INTEREST: None}

\section{ACKNOWLEDGEMENTS: None}

\section{WHY THIS PAPER IS IMPORTANT TO PAEDIATRIC DENTISTS:}

Burkitt lymphoma is a rare entity resulting in delayed diagnosis causing worse prognosis

Given the early age of onset and the osteolytic character of the condition dental germs and its anatomical landmarks can be affected.

AUTHOR CONTRIBUTIONS:D.G. conceived the ideas, W.D.C. and D.G. collected the data, W.D.C. did the writing, D.G. led the writing, C.P., G.V., M.B., T.T. and A.U. did the editing 


\section{REFERENCES}

1. Ogwang MD, Bhatia K, Biggar RJ, Mbulaiteye SM. Incidence and geographic distribution of endemic Burkitt lymphoma in northern Uganda revisited. Int $J$ cancer . 2008;123(11):2658-2663. doi:10.1002/ijc. 23800

2. Kalisz K, Alessandrino F, Beck R, et al. An update on Burkitt lymphoma: a review of pathogenesis and multimodality imaging assessment of disease presentation, treatment response, and recurrence.Insights Imaging . 2019;10(1):56. doi:10.1186/s13244-019-0733-7

3. Mbulaiteye SM, Anderson WF, Ferlay J, et al. Pediatric, elderly, and emerging adult-onset peaks in Burkitt's lymphoma incidence diagnosed in four continents, excluding Africa. Am J Hematol . 2012;87(6):573-578. doi:10.1002/ajh.23187

4. Morton LM, Wang SS, Devesa SS, Hartge P, Weisenburger DD, Linet MS. Lymphoma incidence patterns by WHO subtype in the United States, 1992-2001. Blood . 2006;107(1):265-276. doi:10.1182/blood-200506-2508

5. Derinkuyu BE, Boyunağa Ö, Öztunalı Ç, et al. Imaging features of Burkitt lymphoma in pediatric patients. Diagn Interv Radiol . 2016;22(1):95-100. doi:10.5152/dir.2015.15211

6. Egan G, Goldman S, Alexander S. Mature B-NHL in children, adolescents and young adults: current therapeutic approach and emerging treatment strategies. Br J Haematol . 2019;185(6):1071-1085. doi:10.1111/bjh.15734

7. Silva TDB, Ferreira CBT, Leite GB, de Menezes Pontes JR, Antunes HS. Oral manifestations of lymphoma: a systematic review.Ecancermedicalscience . 2016;10:665. doi:10.3332/ecancer.2016.665

8. Balasubramaniam R, Goradia A, Turner LN, et al. Burkitt lymphoma of the oral cavity: an atypical presentation. Oral Surg Oral Med Oral Pathol Oral Radiol Endod . 2009;107(2):240-245. doi:10.1016/j.tripleo.2008.09.008

9. Mbulaiteye SM, Biggar RJ, Bhatia K, Linet MS, Devesa SS. Sporadic childhood Burkitt lymphoma incidence in the United States during 1992-2005. Pediatr Blood Cancer . 2009;53(3):366-370. doi: $10.1002 /$ pbc. 22047

10. White SC, Pharoah MJ. Oral Radiology: Principles and Interpretation . Fifth edit. Mosby; 2004.

11. Khanna G, Sato Y, Smith RJH, Bauman NM, Nerad J. Causes of facial swelling in pediatric patients: correlation of clinical and radiologic findings. Radiographics . 2006;26(1):157-171. doi:10.1148/rg.261055050

12. Anil Govindrao Ghom. Basic Oral Radiology . First edit. Jaypee brothers medical publishers Ltd; 2014.

13. Storck K, Brandstetter M, Keller U, Knopf A. Clinical presentation and characteristics of lymphoma in the head and neck region. Head Face Med . 2019;15(1):1. doi:10.1186/s13005-018-0186-0

14. Minard-Colin V, Aupérin A, Pillon M, et al. Rituximab for high-risk, mature B-cell non-Hodgkin's lymphoma in children. $N$ Engl J Med . 2020;382(23):2207-2219. doi:10.1056/NEJMoa1915315

15. Murphy SB. Classification, staging and end results of treatment of childhood non-Hodgkin's lymphomas: dissimilarities from lymphomas in adults. Semin Oncol . 1980;7(3):332-339.

16. Boon LC, Nik-Hussien NN. Burkitt's lymphoma in the mandible-a case report. Br J Oral Maxillofac Surg . 1987;25(5):410-414. doi:10.1016/0266-4356(87)90092-1

17. Rodrigues-Fernandes CI, Pérez-de-Oliveira ME, Aristizabal Arboleda LP, et al. Clinicopathological analysis of oral Burkitt's lymphoma in pediatric patients: A systematic review. Int $J$ Pediatr Otorhinolaryngol . 2020;134:110033. 
18. Palmer RM, Lumsden AG. Development of periodontal ligament and alveolar bone in homografted recombinations of enamel organs and papillary, pulpal and follicular mesenchyme in the mouse. Arch Oral Biol . 1987;32(4):281-289. doi:10.1016/0003-9969(87)90022-7

19. Curtis AB. Childhood leukemias: osseous changes in jaws on panoramic dental radiographs. J Am Dent Assoc . 1971;83(4):844-847. doi:10.14219/jada.archive.1971.0405

20. Hanazawa T, Kimura Y, Sakamaki H, Yamaguchi A, Nagumo M, Okano T. Burkitt's lymphoma involving the mandible: report of a case and review of Japanese cases. Oral Surg Oral Med Oral Pathol Oral Radiol Endod . 1998;85(2):216-220. doi:10.1016/s1079-2104(98)90429-2

21. Wood RE, Nortjé CJ, Hesseling P, Mouton S. Involvement of the maxillofacial region in African Burkitt's lymphoma in the Cape Province and Namibia. Dentomaxillofac Radiol . 1988;17(1):57-60. doi:10.1259/dmfr.1988.0007

22. Dunfee BL, Sakai O, Pistey R, Gohel A. Radiologic and pathologic characteristics of benign and malignant lesions of the mandible.Radiogr a Rev Publ Radiol Soc North Am Inc . 2006;26(6):1751-1768. doi:10.1148/rg.266055189

23. Satheesan E, Tamgadge S, Tamgadge A, Bhalerao S, Periera T. Histopathological and Radiographic Analysis of Dental Follicle of Impacted Teeth Using Modified Gallego's Stain. J Clin Diagn Res . 2016;10(5):ZC106-11. doi:10.7860/JCDR/2016/16707.7838

24. El-Naggar AK, Chan JK, Grandis JR, Takata T, Slootweg PJ. WHO Classification of Head and Neck Tumours . 4th editio. International Agency for Research on Cancer; 2017.

25. Sandlund JT, Pui C-H, Zhou Y, et al. Effective treatment of advanced-stage childhood lymphoblastic lymphoma without prophylactic cranial irradiation: results of St Jude NHL13 study. Leukemia . 2009;23(6):11271130. doi: $10.1038 /$ leu.2008.400

26. Gerrard M, Cairo MS, Weston C, et al. Excellent survival following two courses of COPAD chemotherapy in children and adolescents with resected localized B-cell non-Hodgkin's lymphoma: results of the FAB/LMB 96 international study. Br J Haematol . 2008;141(6):840-847. doi:10.1111/j.1365-2141.2008.07144.x

27. Avril L, Lombardi T, Ailianou A, et al. Radiolucent lesions of the mandible: a pattern-based approach to diagnosis. Insights Imaging . 2014;5(1):85-101. doi:10.1007/s13244-013-0298-9

Figure 1. Intra-oral image of friction lesion at the level of the left cheek (A ). Oral lesion after biopsy and chemotherapy (B).

Figure 2. Panoramic radiograph with an osteolytic lesion at the left corner of the jaw. Mark the absence of a cortical border around the follicular sac. (compare with the contralateral side)

Figure 3 . Coronal PET-CT image showing various abdominal metastases, mark the femoral hyperintensity (A ). Axial MRI image showing the lesion of the left jaw (B ). Axial CT image of a mass in the left masseter muscle region, fixed on the ascending mandibular ramus with osteolysis of the bony tissue $(\mathbf{C})$.

Figure 4 . Histopathology of an incisional biopsy of the mucosa of the jaw (A ). H\&E stain shows the lesion is composed of a diffuse proliferation of medium-sized, monomorphic blastoid cells with round vesicular to hyperchromatic nuclei. The nuclei contained small nucleoli and numerous mitotic figures were present. Between the cells there are tingible body macrophages giving rise to a starry sky appearance. The cells express CD20 (B ), CD10 (C), but no BCL2 (D ). The proliferation index is very high (E), and there is nuclear expression of MYC (F ) in the majority of cells, due to a MYC-rearrangement, proven by FISH. All images are taken at 400X magnification, except $\mathbf{A}$, taken at 200X. 

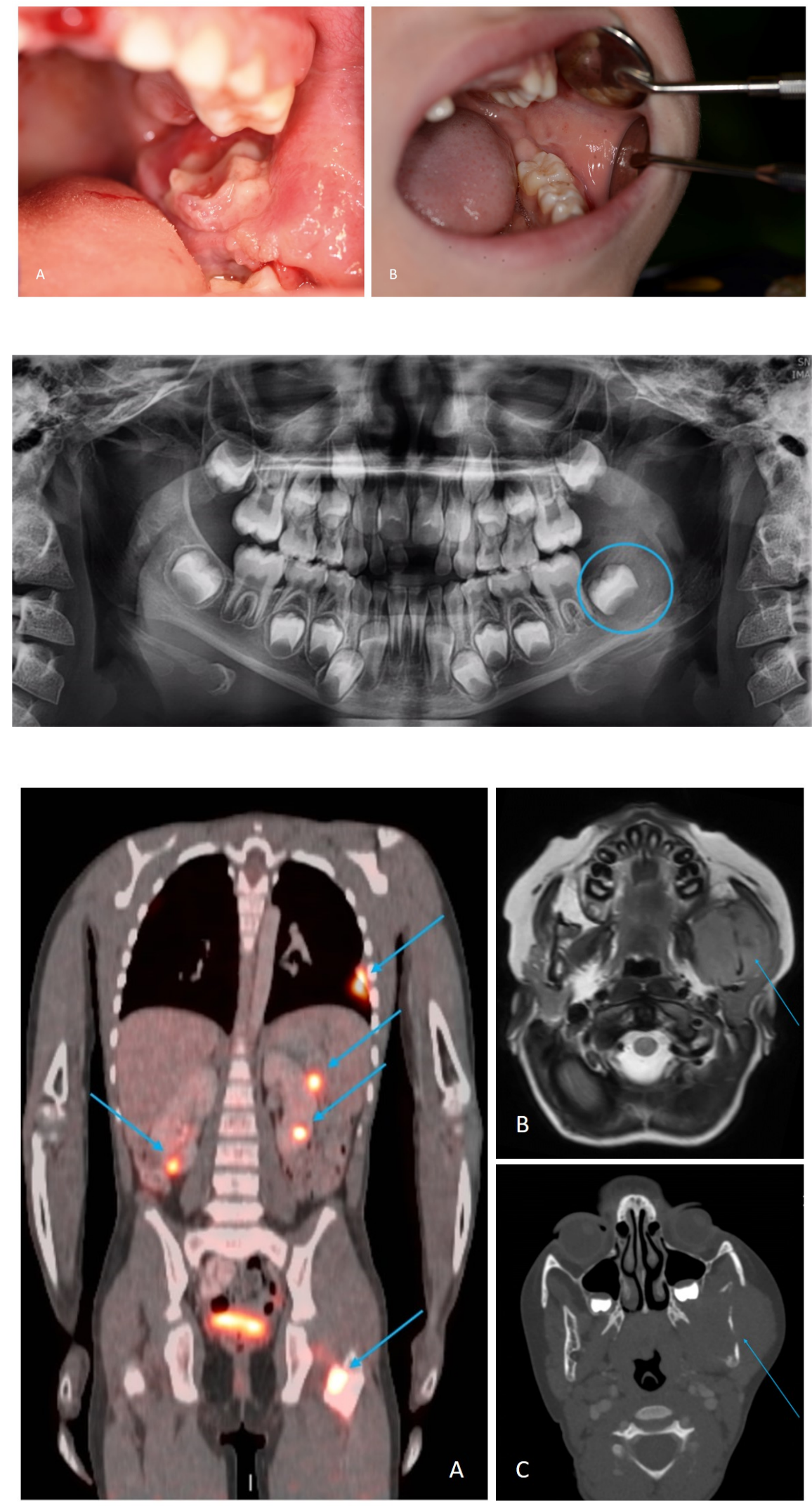

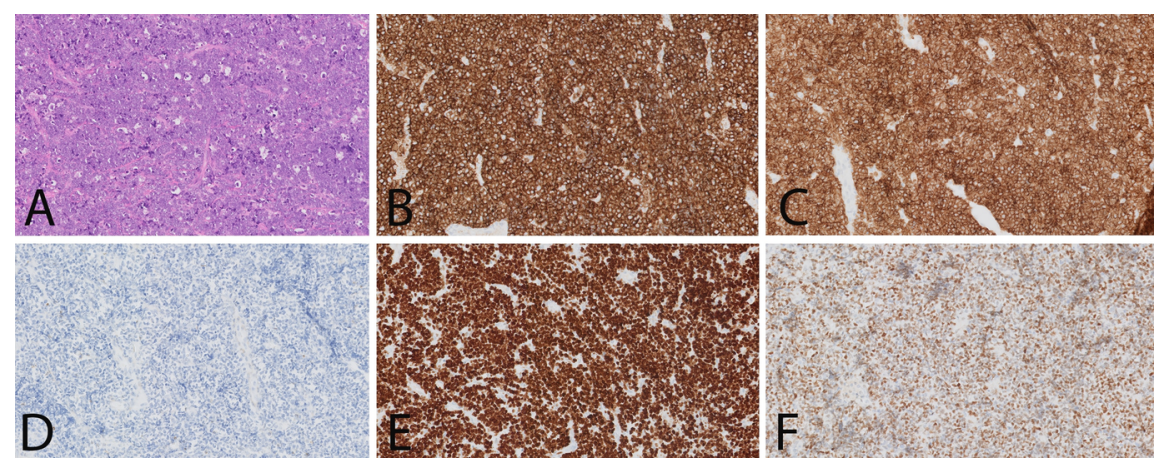